\title{
Liter per Hour
}

National Cancer Institute

\section{Source}

National Cancer Institute. Liter per Hour. NCI Thesaurus. Code C69160.

A non-SI unit of volumetric flow rate defined as the rate at which one liter of matter crosses a given surface during the period of time equal to one hour. 